\title{
Development and clinical validation of a grading system for pituitary adenoma consistency
}

\author{
Martin J. Rutkowski, MD, ${ }^{1}$ Ki-Eun Chang, MD, ${ }^{1}$ Tyler Cardinal, BS, ${ }^{1}$ Robin Du, BS, ${ }^{1}$ \\ Ali R. Tafreshi, MD, ${ }^{1}$ Daniel A. Donoho, MD, ${ }^{1}$ Andrew Brunswick, MD, ${ }^{1}$ Alexander Micko, MD, PhD, ${ }^{1}$ \\ Chia-Shang J. Liu, MD, PhD, ${ }^{3}$ Mark S. Shiroishi, MD, ${ }^{3}$ John D. Carmichael, MD, ${ }^{2}$ and \\ Gabriel Zada, MD'
}

1Department of Neurological Surgery, ${ }^{2}$ Division of Endocrinology, Department of Internal Medicine, and ${ }^{3}$ Department of Radiology, Keck Medical Center, University of Southern California, Los Angeles, California

OBJECTIVE Pituitary adenoma (PA) consistency, or texture, is an important intraoperative characteristic that may dictate operative dissection techniques and/or instruments used for tumor removal during endoscopic endonasal approaches (EEAs). The impact of PA consistency on surgical outcomes has yet to be elucidated.

METHODS The authors developed an objective 5-point grading scale for PA consistency based on intraoperative characteristics, including ease of tumor debulking, manipulation, and instrument selection, ranging from cystic/hemorrhagic tumors (grade 1) to calcified tumors (grade 5). The proposed grading system was prospectively assessed in 306 consecutive patients who underwent an EEA for PAs, and who were subsequently analyzed for associations with surgical outcomes, including extent of resection (EOR) and complication profiles.

RESULTS Institutional database review identified 306 patients who underwent intraoperative assessment of PA consistency, of which $96 \%$ were macroadenomas, $70 \%$ had suprasellar extension, and $44 \%$ had cavernous sinus invasion (CSI). There were 214 (69.9\%) nonfunctional PAs and 92 functional PAs (31.1\%). Distribution of scores included 15 grade 1 tumors (4.9\%), 112 grade 2 tumors (36.6\%), 125 grade 3 tumors (40.8\%), 52 grade 4 tumors (17\%), and 2 grade 5 tumors (0.7\%). Compared to grade $1 / 2$ and grade 3 PAs, grade 4/5 PAs were significantly larger (22.5 vs 26.6 vs $27.4 \mathrm{~mm}$, $p<0.01)$, more likely to exhibit CSI ( $39 \%$ vs $42 \%$ vs $59 \%, p<0.05)$, and trended toward nonfunctionality $(67 \%$ vs $68 \%$ vs $82 \%, p=0.086$ ). Although there was no association between PA consistency and preoperative headaches or visual dysfunction, grade 4/5 PAs trended toward preoperative $(p=0.058)$ and postoperative panhypopituitarism $(p=0.066)$. Patients with preoperative visual dysfunction experienced greater improvement if they had a grade 1/2 PA $(p<0.05)$. Intraoperative CSF leaks were noted in $32 \%$ of cases and were more common with higher-consistency-grade tumors ( $p$ $=0.048)$, although this difference did not translate to postoperative CSF leaks. Gross-total resection (\%) was more likely with lower PA consistency score as follows: grade $1 / 2(60 \%)$, grade $3(50 \%)$, grade $4 / 5(44 \% ; p=0.045)$. Extracapsular techniques were almost exclusively performed in grade 4/5 PAs. Assignment of scores showed low variance and high reproducibility, with an intraclass correlation coefficient of 0.905 (95\% $\mathrm{Cl} 0.815-0.958)$, indicating excellent interrater reliability.

CONCLUSIONS These findings demonstrate clinical validity of the proposed intraoperative grading scale with respect to PA subtype, neuroimaging features, EOR, and endocrine complications. Future studies will assess the relation of PA consistency to preoperative MRI findings to accurately predict consistency, thereby allowing the surgeon to tailor the exposure and prepare for varying resection strategies.

https://thejns.org/doi/abs/10.3171/2020.4.JNS193288

KEYWORDS pituitary surgery; adenoma; consistency; endoscopic; transsphenoidal; extent of resection; recurrence

ABBREVIATIONS CSI = cavernous sinus invasion; $\mathrm{DI}=$ diabetes insipidus; $\mathrm{EEA}=$ endoscopic endonasal approach; $\mathrm{EOR}=$ extent of resection; GH = growth hormone; GTR = gross-total resection; IGF = insulin-like growth factor; PA = pituitary adenoma; USC = University of Southern California.

SUBMITTED December 10, 2019. ACCEPTED April 3, 2020.

INCLUDE WHEN CITING Published online June 5, 2020; DOI: 10.3171/2020.4.JNS193288. 
$\mathrm{P}$ ITUITARY adenomas (PAs) comprise $10 \%-25 \%$ of all intracranial neoplasms, with a prevalence of $16.9 \%$ in autopsy studies. ${ }^{1}$ These lesions often invade the clivus and cavernous sinuses, or extend into the suprasellar cistern, complicating efforts to achieve gross-total resection (GTR). While PAs have traditionally been resected through microscopic approaches, continued incorporation and optimization of endoscopic techniques has proven safe and effective for the resection of PAs, even for large and invasive lesions. ${ }^{2,3}$

Beyond endocrine syndromes secondary to hormonal hypersecretion noted in functional PAs, these tumors may become symptomatic due to visual deficits, cranial neuropathy, hypopituitarism, and headaches, all through local mass effect. Extent of resection (EOR) has long proven a durable metric for clinical outcomes that include rates of recurrence and improvement or resolution of visual compromise and endocrinopathy. ${ }^{4}$ Although tumor size, invasion, and biology have been correlated with EOR, the impact of tumor consistency remains unclear. Most PAs are of intermediate texture or consistency, and are generally resectable with dissection, curettage, and aspiration; however, up to $15 \%$ of PAs may be firm, fibrous, and unamenable to traditional manipulation and removal, requiring expanded endonasal or open, transcranial approaches for resection. ${ }^{5}$ As the armamentarium of open and endonasal approaches increases, the impact of tumor consistency on EOR and approach selection has yet to be elucidated; a priori knowledge of tumor consistency may allow surgeons to better plan effective approaches for achieving GTR and optimizing patient safety. For instance, softer tumors may be preferentially resected via minimally invasive approaches, whereas firmer and/or calcified PAs may require alternative operative strategies including extended approaches, ultrasonic aspirators, extracapsular dissection, or rarely, open craniotomy.

Preoperative prediction of PA consistency could augment operative planning and approach selection, but remains an imprecise effort that suffers from conflicting results in the radiology literature and lack of an established, universally applicable and objective grading system. ${ }^{6-8}$ For these reasons, we performed a prospective assessment of intraoperative consistency in a cohort of 306 consecutive functional and nonfunctional PAs, encompassing a continuous spectrum of tumoral texture and consistency based on 5 subtypes. This practical 5-point grading system relies on features such as cystic and liquid contents, ability to resect with blunt versus sharp dissection, pseudocapsular maneuverability and tumor descent from the suprasellar cistern, and the presence of calcifications with the need for powered instrumentation for resection. We then assessed the clinical utility of our proposed consistency grading scale by determining its impact on preoperative symptomatology, local invasion or extension, perioperative complications, EOR, pathology, and postoperative complications.

\section{Methods \\ Patient Selection}

A retrospective review of a prospectively collected database was conducted following IRB approval at the Keck
School of Medicine. We reviewed a retrospectively collected database of all endoscopic endonasal surgeries performed by the senior author (G.Z.) at Los Angeles County + University of Southern California (USC) Medical Center and Keck Hospital of USC between September 2012 and April 2019. Three hundred six patients with PAs were prospectively assigned a tumor consistency score, in addition to cataloged data including patient demographics, baseline endocrinopathies, tumor characteristics (including size, functionality, invasion, extension, and pathology), perioperative complications, operative outcomes (including EOR and recurrence), postoperative complications, and clinical outcomes (including improvement or worsening of endocrinopathy, visual deficits, and headaches).

\section{Consistency Score Assignment}

A 5-point grading scale was developed to assess intraoperative consistency of PAs (Table 1), ranging from softest (grade 1) to firmest (grade 5). The authors aimed to create a universally applicable, practical, and objective scale. Assessment is based on ease of PA manipulation and resectability, with a focus on factors such as texture, capsular integrity, descent from the suprasellar cistern, and mechanism of debulking and resection (e.g., suction, mechanical debulking, curettage, and piecemeal vs en bloc resection).

Grade 1 PAs are cystic and/or hemorrhagic tumors that are mostly liquified and amenable to suction alone. Grade 2 PAs are soft, solid tumors that require minimal mechanical manipulation for resection and are freely suckable. Grade 3 PAs require mechanical debulking to establish planes and free up tumor fragments, but can be resected through a combination of suction and curettage; when present, suprasellar tumor fragments readily descend into the sella for removal. Grade 4 PAs have a firm consistency unamenable to suction and necessitate mechanical manipulation to establish planes for resection, which requires curettage or mechanical debulking; suprasellar components do not descend readily, and extracapsular dissection is typically required. Grade 5 PAs are extremely firm and/ or calcified and incapable of curettage, requiring extensive sharp, mechanical, or en bloc removal.

Video 1 highlights the spectrum of PA consistencies; this intraoperative video shows tumors ranging from grade $1-5$, including illustrative features that determine grade and corresponding surgical and technical nuances for dissection and resection.

VIDEO 1. Clip showing PA resections highlighting features of consistency grades 1-5. Copyright Martin Rutkowski. Published with permission. Click here to view.

\section{Neuroimaging Evaluation}

All patients underwent preoperative MRI to assess tumor size, presence of cavernous sinus invasion, infrasellar invasion, suprasellar extension, sphenoid anatomy, carotid anatomy, presence of residual pituitary gland, and position of the optic chiasm and nerves. Subsequently, patients underwent postoperative MRI at 3 months to assess for EOR, and then again annually to rule out tumor progression or recurrence. Tumor size was defined as the maximal diam- 
TABLE 1. A proposed 5-point scale for pituitary adenoma consistency

\begin{tabular}{clc}
\hline $\begin{array}{c}\text { Consistency } \\
\text { Grade }\end{array}$ & \multicolumn{1}{c}{ Description } & Overall Distribution \\
\hline 1 & Cystic or hemorrhagic tumor consistency & $4.9 \%$ \\
\hline 2 & Soft tumor consistency; freely suckable tumor; minimal curettage required & $36.6 \%$ \\
\hline 3 & $\begin{array}{c}\text { Average tumor consistency; partially suckable tumor, requires some curettage or mechani- } \\
\text { cal debulking; tumor readily descends from suprasellar space }\end{array}$ & $40.8 \%$ \\
\hline 4 & $\begin{array}{l}\text { Firm tumor consistency; not suckable, curettage or mechanical debulking required; tumor } \\
\text { does not readily descend from suprasellar space; extracapsular technique typically } \\
\text { required }\end{array}$ & $17 \%$ \\
\hline 5 & Extremely firm or calcified tumor; not curettable, requires sharp or en bloc removal & $0.7 \%$ \\
\hline
\end{tabular}

eter in either the anteroposterior, lateral, or rostrocaudal dimension. Parasellar invasion into the cavernous sinuses was assessed according to the Knosp classification, with grades 3 and 4 considered definite invasion. ${ }^{9}$ Suprasellar extension was assessed by the presence of tumor above a line extending from the tuberculum to the dorsum sella, and infrasellar invasion as tumor infiltration beyond the sellar floor into the sphenoid sinus or into the clivus. Recurrence was defined as growth of known residual tumor or the reappearance of tumor following previously radiologically documented GTR.

\section{Endocrinological Evaluation}

A full pituitary panel was obtained preoperatively, including prolactin, thyroid-stimulating hormone, free T4, T3, adrenocorticotropic hormone, random cortisol, follicle-stimulating hormone, luteinizing hormone, testosterone, growth hormone (GH), and insulin-like growth factor-1 (IGF-1). Further testing of patients with suspected functional adenomas included 24-hour urinary-free cortisol and/or midnight salivary cortisol levels, and/or dexamethasone suppression testing for Cushing's disease; repeat GH and IGF-1 levels for acromegaly; and repeat prolactin levels for suspected prolactinomas. Follow-up endocrinological testing is regularly performed 6 weeks postoperatively to assess for biochemical remission in functional adenomas and for improvement or worsening of hypopituitarism, and repeated at 3 months postoperatively if needed.

\section{Statistical Analysis}

Following the assignment of an intraoperative consistency score, scores were analyzed as a continuous variable against patient demographic data (age, sex, presenting symptoms), tumor characteristics (size, invasion, functionality, pathology), complication profiles, EOR, and long-term follow-up data, including recurrence, endocrine status, and resolution of preoperative signs and symptoms to determine the significance of consistency.

Means were compared using the independent samples t-test when the variable was normally distributed, and the Wilcoxon rank-sum test (Mann-Whitney U-test) when the distribution was not normal. Categorical variables were analyzed using Pearson's chi-square analysis or Fisher's exact test. Correlations were determined using Spearman's correlation coefficient test for nonnormally distrib- uted variables or the Pearson correlation coefficient test for normally distributed variables. An ANOVA with Tamhane's $\mathrm{T} 2$ post hoc testing was used to assess the means between 3 or more variables.

In an effort to assess the validity and reproducibility of the scale, four independent postgraduate neurosurgeons (M.J.R., A.B., A.M., and G.Z.) assigned consistency scores for tumors in 20 patients by watching intraoperative video of PA dissection and resection. Subsequently, reliability analysis was performed by calculating an intraclass correlation coefficient and Cronbach alpha statistic to determine interrater reliability. These tests were chosen given our use of four nonunique, independent scoring observers assigning adenoma consistency scores as a continuous variable. Statistical analysis was performed using SPSS statistical software (version 24.0, IBM Corp.). A 2-tailed p value $<0.05$ was considered significant.

\section{Results}

\section{Patient Demographics and Presenting Signs and Symptoms}

Between the years 2012-2019, 306 patients were prospectively assigned a PA consistency grade while undergoing endonasal endoscopic transsphenoidal resection (Table 2). There were 175 female patients (57\%) and 131 male patients $(43 \%)$, with a mean age of 52 years (range 16-92 years). Common presenting signs and symptoms included headaches in $63 \%$ of patients, visual dysfunction in $51 \%$, visual field cuts in $29 \%$, and ophthalmoplegia in $7 \%$. Endocrinopathy included partial hypopituitarism in $29 \%$ of patients, panhypopituitarism in $10 \%$, and diabetes insipidus (DI) in $1 \%$. Hypersecretory syndromes included hyperprolactinemia in $21 \%$ of patients, acromegaly in $14 \%$, and Cushing's disease in 5\%.

\section{Tumor Characteristics and Consistency Scores}

The majority of tumors (96\%) were macroadenomas (Table 2), with $70 \%$ showing suprasellar extension and $44 \%$ showing cavernous sinus invasion. Knosp scores were available for 92 patients: $14.1 \%$ Knosp grade $0,31.5 \%$ Knosp grade 1, 16.3\% Knosp grade 2, 22.8\% Knosp grade 3 , and $15.2 \%$ Knosp grade 4. Infrasellar invasion through the sellar floor into the sphenoid sinus and/or clivus was seen in 16\% of patients. There were 214 nonfunctional PAs (69.9\%), 36 somatotrophs (11.8\%), 14 prolactinomas 
TABLE 2. Patient, tumor, and outcome characteristics stratified by PA consistency grade

\begin{tabular}{|c|c|c|c|}
\hline \multirow[b]{2}{*}{ Variable } & \multicolumn{3}{|c|}{ Consistency Grade } \\
\hline & 1 or 2 & 3 & 4 or 5 \\
\hline \multicolumn{4}{|l|}{ Demographics, presenting signs and symptoms } \\
\hline Patients, $\mathrm{n}(\%)$ & $127(41.5)$ & $125(40.8)$ & $54(17.6)$ \\
\hline Females, n (\%) & $84(66.1)$ & $61(48.8)$ & $30(55.6)$ \\
\hline Mean age (range), yrs & $51.1(16-92)$ & $52.1(19-80)$ & $51.8(28-89)$ \\
\hline Preop headache, $\mathrm{n}(\%)$ & $78(61.4)$ & $80(64)$ & $34(63)$ \\
\hline Preop vision loss, $n(\%)$ & $62(48.8)$ & $62(49.6)$ & $31(57.4)$ \\
\hline Preop hypopituitarism, $\mathrm{n}(\%)$ & $13(10.2)$ & $13(10.4)$ & $4(7.4)$ \\
\hline \multicolumn{4}{|c|}{ Tumor characteristics and consistency scores, $\mathrm{n}(\%)$} \\
\hline Macroadenomas & $120(94.5)$ & $120(96.0)$ & $53(98.1)$ \\
\hline Suprasellar extension & $83(65.4)$ & $90(72.0)$ & $40(74.1)$ \\
\hline Cavernous sinus invasion & $50(39.4)$ & $53(42.4)$ & $32(59.3)$ \\
\hline Infrasellar invasion & $17(13.4)$ & $20(16.0)$ & $12(22.2)$ \\
\hline Functional & $42(33.1)$ & $40(32.0)$ & $10(18.5)$ \\
\hline Nonfunctional & $85(66.9)$ & $85(68.0)$ & $44(81.5)$ \\
\hline Mean tumor size $\pm \mathrm{SD}, \mathrm{mm}$ & $22.5 \pm 10.5$ & $26.6 \pm 11.2$ & $27.4 \pm 11.8$ \\
\hline \multicolumn{4}{|c|}{ Overall operative outcomes and complication profiles } \\
\hline Repeat resection, $\mathrm{n}(\%)$ & $19(15.0)$ & $13(10.4)$ & $12(22.2)$ \\
\hline Extended endoscopic approach, $n(\%)$ & $3(2.4)$ & $4(3.2)$ & $4(7.4)$ \\
\hline Surgery performed at Keck Hospital, n (\%) & $94(74.0)$ & $62(49.6)$ & $27(50.0)$ \\
\hline Known EOR, GTR (\%)/STR (\%) & $65(60) / 44(40)$ & $51(50) / 51(50)$ & $21(44) / 27(56)$ \\
\hline Recurrence following GTR, n & 1 & 1 & 0 \\
\hline Progression of known residual disease, $n$ & 8 & 10 & 5 \\
\hline Intraop CSF leak, n (\%) & $34(26.8)$ & $40(32.0)$ & $24(44.4)$ \\
\hline Postop CSF leak, n (\%) & $7(5.5)$ & $5(4.0)$ & $5(9.3)$ \\
\hline Hyponatremia, n (\%) & $10(7.9)$ & $7(5.6)$ & $8(14.8)$ \\
\hline Transient DI, n (\%) & $11(8.7)$ & $15(12.0)$ & $5(9.3)$ \\
\hline Permanent DI, n (\%) & $1(0.8)$ & $6(4.8)$ & 0 \\
\hline Meningitis, $\mathrm{n}(\%)$ & $1(0.8)$ & $2(1.6)$ & 0 \\
\hline Sinusitis, $\mathrm{n}(\%)$ & $4(3.1)$ & $2(1.6)$ & 0 \\
\hline Epistaxis, n (\%) & $6(4.7)$ & $6(4.8)$ & $3(5.6)$ \\
\hline Improvement in preop headache, $\mathrm{n}(\%)$ & $45 / 78(57.7)$ & $57 / 80(71.3)$ & $22 / 34(64.7)$ \\
\hline Improvement in preop visual deficits, $\mathrm{n}(\%)$ & $48 / 62(77.4)$ & $48 / 62(77.4)$ & $16 / 31(51.6)$ \\
\hline Improvement in hormonal axes, $\mathrm{n}(\%)$ & $48(37.8)$ & $33(26.4)$ & $10(18.5)$ \\
\hline
\end{tabular}

STR = subtotal resection.

(4.6\%), 14 corticotrophs (4.6\%), 14 silent corticotrophs (4.6\%), 7 mammosomatotrophs (2.3\%), 2 other plurihormonal tumors $(0.7 \%)$, and 1 thyrotroph PA $(0.3 \%)$. There was no correlation between pathologic subtype and tumor consistency. Mean tumor size was $25 \pm 11.2 \mathrm{~mm}$ (range 5-70 mm). While data were limited for grade 5 tumors $(\mathrm{n}=2)$, larger tumors on average had higher consistency scores (grade $1=20.4 \mathrm{~mm}$, grade $2=22.8 \mathrm{~mm}$, grade $3=$ $26.6 \mathrm{~mm}$, grade $4=27.8 \mathrm{~mm}$, grade $5=16 \mathrm{~mm}, \mathrm{p}<0.01$, ANOVA).

\section{Overall Operative Outcomes and Complication Profiles}

Two-hundred sixty-two patients (86\%) underwent primary resection, and 44 patients (14\%) underwent repeat re- section. Eleven patients (3.6\%) underwent an extended endoscopic approach. Of the 44 patients who underwent prior resection of their adenoma with evidence of symptomatic recurrence, 37 patients had 1 prior operation, 5 had 2 prior operations, and 2 had 3 prior operations. Operations were performed at the Keck Hospital in $60 \%$ of cases, while $40 \%$ were performed at the affiliated LAC + USC Medical Center. Follow-up neuroimaging data were available for 259 patients (85\%): there were 137 GTRs $(53 \%)$ and 122 subtotal resections (47\%). Two patients showed evidence of recurrence following GTR at 4 and 19 months, while 23 patients showed evidence of progression of known residual disease at a mean of 19 months.

There were no perioperative deaths or internal carotid 
artery injuries in this series. Intraoperative CSF leak was noted in 32\% of cases, and postoperative CSF leaks in 17 patients $(5.6 \%)$. Hyponatremia was seen in $8.2 \%$, transient DI in $10 \%$, permanent DI in $2.3 \%$, meningitis in $1 \%$, sinusitis in $2 \%$, and epistaxis in $5 \%$.

Among patients with preoperative headaches, $64.6 \%$ showed improvement and $32.8 \%$ demonstrated no change. Preoperative visual deficits improved in $72 \%$ of patients, worsened in $0.6 \%$, and stayed stable in $24.5 \%$. Postoperatively, 2 patients $(0.6 \%)$ had new headaches and 2 patients $(0.6 \%)$ had new visual deficits. Improvement in one or more hormonal axes was observed in $30 \%$ of patients, stable pituitary function or stable hypopituitarism in 53\%, new partial hypopituitarism in 4\%, and new panhypopituitarism in $12 \%$ of patients.

\section{Impact of Tumor Consistency on Clinical Presentation, Adenoma Behavior, and Surgical Outcomes}

The overall distribution of consistency scores included 15 grade 1 tumors (4.9\%), 112 grade 2 tumors (36.6\%), 125 grade 3 tumors $(40.8 \%), 52$ grade 4 tumors $(17 \%)$, and 2 grade 5 tumors $(0.7 \%$; Fig. 1 , Table 1$)$.

Male patients tended to have higher-consistency-grade tumors, with a mean consistency grade of 2.85 versus 2.62 for female patients $(\mathrm{p}=0.016$, $\mathrm{t}$-test). Tumors extending into the suprasellar cistern, invading the cavernous sinuses, clivus, or through the sellar floor into the sphenoid had a higher consistency grade ( 2.77 vs $2.48, \mathrm{p}=0.020$, t-test). This was also true when specifically examining cavernous sinus invasion ( 2.83 vs $2.63, \mathrm{p}=0.037$, t-test), although there did not appear to be an association with degree of invasion as determined by Knosp grade.

Intraoperative CSF leaks were more common with higher-consistency-grade tumors ( 2.86 vs $2.66, \mathrm{p}=0.048$, $\mathrm{t}$-test), although this did not remain true for postoperative CSF leaks $(\mathrm{p}=0.592, \mathrm{t}$-test). There was no association between tumor consistency and preoperative symptoms including headaches, visual dysfunction, visual field cuts, and endocrinopathy, with the exception of panhypopituitarism. Patients who presented with panhypopituitarism had a clinical trend toward higher-consistency-grade tumors (2.97 vs $2.69, \mathrm{p}=0.058$, t-test). Furthermore, patients with higher consistency PAs also showed a trend toward new panhypopituitarism (2.81 vs $2.52, \mathrm{p}=0.066$, t-test). There was also a trend toward functional tumors being lower consistency grade (2.59 vs $2.77, \mathrm{p}=0.086$, t-test). Patients with lower-consistency-grade tumors were more likely to undergo GTR: the mean consistency score associated with GTR was 2.62 versus 2.83 for tumors that underwent STR ( $p=0.045$, t-test), although this association dropped out of the multivariate model when controlling for size and cavernous sinus invasion.

We performed further analysis by combining consistency score subgroups into three cohorts based on surgical technique required. The first group (previously grades 1 and 2) included tumors that can be resected via simple suction; the second group (grade 3) included tumors resected through mechanical debulking and suction; and the third group (grades 4 and 5) included tumors unamenable to suction that require mechanical debulking. When analyzed by group, firmer tumors tended to be larger (22.5 vs

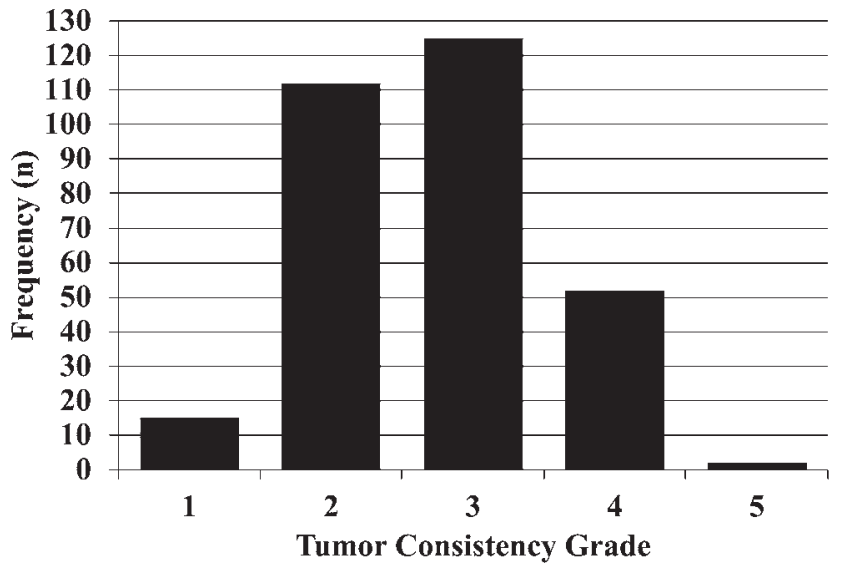

FIG. 1. Graph demonstrating the frequencies of each tumor consistency grade included in the study.

26.6 vs $27.4 \mathrm{~mm}, \mathrm{p}<0.01$, ANOVA) and more invasive ( $39 \%$ vs $42 \%$ vs $59 \%$ invasive, $p<0.05$, ANOVA). Finally, patients with preoperative visual dysfunction experienced greater improvement in their signs and symptoms if they had a lower-consistency-grade (1-2) tumor $(\mathrm{p}<0.05$, ANOVA), although this lost significance when controlling for tumor size (micro- or macroadenoma).

\section{Reproducibility and Reliability of PA Consistency Grading}

Interrater reliability and reproducibility of PA consistency scores was high. When examining the overall variance in scores between four observers over 20 assigned consistency grades, the intraclass correlation coefficient was 0.905 (95\% CI 0.815-0.958), indicating excellent interrater agreement and low interrater variance. Furthermore, the Cronbach alpha statistic was 0.908 , indicating high reliability of the scoring system among the four observers.

\section{Discussion}

The expanding armamentarium of minimally invasive skull base techniques, including direct and extended endonasal endoscopic approaches, requires a nuanced consideration of patient and tumor factors that favor one approach versus another. In addition to approach angle, local tumor invasion, proximity to critical neurovascular structures, and reconstruction and closure options, tumor consistency significantly influences the ability to dissect, manipulate, and resect skull base tumors such as PAs..$^{10-12}$ Instrumentation needs can also vary based on tumor consistency, further corroborating the principle that safe and effective tumor resection depends equally on approach selection and resection strategy. In this study, retrospective analysis of prospectively assigned OPA consistency scores show a clear association with patient sex, tumor diameter, degree of extrasellar invasiveness or extension, and degree of resection.

Our group has already developed, validated, and prospectively applied a meningioma consistency score in an effort to standardize and simplify this universal tumor 
metric; we found a correlation between tumor vascularity, size, and skull base location, as well as operative duration and EOR..$^{13,14}$ However, development of a clinically validated grading scale for consistency has not been previously reported for PAs. In this paper we introduce and implement a 5-point grading scale for PA consistency with the intent to provide an easily applicable, universal, and clinically relevant scoring method to standardize grading.

Our consistency grading scale specifically focuses on the unique anatomical features of PAs, including extrasellar invasion/extension and interface with the pituitary gland, which influence operative strategy and instrumentation required for each consistency score. This can be particularly vital for functional PAs in which aggressive resection provides the best means of achieving biochemical remission. For example, proper dissection of grade 4 lesions involves identification of the pseudocapsule, a defining trait of grade 4 consistency, to properly develop a plane between tumor and gland and allow for extracapsular dissection. This feature has been repeatedly correlated with biochemical remission in functional adenomas..$^{15-19}$ Our patient cohort demonstrated a wide variety of presenting signs and symptoms, although consistency did not appear to clearly correlate with any specific symptoms preoperatively. While present in a majority of patients, neither headaches nor the presence of visual dysfunction correlated with consistency. Given that many of the deleterious effects of expanding PAs are related to mass effect on adjacent structures, we surmised that firmer tumors might cause more severe deficits at presentation, but this appeared true primarily for hypopituitarism. Patients who presented with panhypopituitarism tended to have firmer tumors; additionally, patients with firmer tumors trended toward an increased incidence of postoperative hypopituitarism. Aggressive en bloc resection of firmer (grade 4 or 5 PAs) may be associated with collateral damage to the normal pituitary gland. The pituitary gland has no means to avoid or escape mass effect from an enlarging adenoma, let alone a firm one, and may explain why higher rates of panhypopituitarism were observed among patients who ultimately proved to have firmer tumors. Manipulation of a firmer tumor also requires more-aggressive maneuvers and resection, potentially placing the residual gland at greater risk of endocrinopathy in patients who develop postoperative panhypopituitarism. This factor may also explain the higher rate of intraoperative CSF leaks noted for firmer tumors. Interestingly, Thotakura and colleagues also suggested a link between firmer tumor consistency and hypopituitarism in their study examining radiological characteristics, which might correlate with intraoperative consistency. ${ }^{20}$

The association between headaches and adenomas remains poorly understood, although our rate of headache improvement (approximately 50\%) matches the rate in the existing literature..$^{21,22}$ Perhaps due to increased intrasellar pressure that creates dural irritation or mass effect, tumors may "decompress" this pressure by invading into nearby structures such as the cavernous sinus or extend into the suprasellar cistern, thus disassociating consistency from headache evolution.

The lack of correlation between consistency and visual dysfunction, including field cuts or cranial neuropathy, may also be explained by the relative mobility of the optic apparatus, and paths of less resistance around and between the optic nerves into the suprasellar, opticocarotid recess, and interpeduncular cisterns. However, the finding that lower-consistency-grade tumors correlate with higher rates of postoperative improvement in vision hints at the role that surgical technique may play in protecting the optic apparatus. Freely suckable tumors, even those with suprasellar extension, allow for distant, indirect decompression of the optic nerves and chiasm by debulking and removing tumor tissue from below, allowing any suprasellar components to freely descend down into the sella where they may also be removed. Higher-consistency-grade tumors may need to be dissected down and off the optic apparatus, thereby increasing the risk of direct manipulation and decreasing the odds of visual improvement.

We found that functional tumors were lower consistency grade, as were those found in women versus men. This may reflect sex differences in the biology of adenomas, or the fact that women were a larger fraction of our overall cohort; as in many PA studies, the more obvious clinical manifestation of hypogonadism in women (dysmenorrhea, amenorrhea) means the incidence of adenomas in women tends to be higher.

Increased size and frequency of invasion in firm tumors may reflect intrinsic differences in tumor biology responsible for their more aggressive behavior; mechanisms of benign tumor invasion are poorly understood, but have been correlated with functionality and the immunological microenvironment surrounding adenomas. ${ }^{23,24}$ Increased collagen content has been proposed as the etiology for firmer meningiomas and possibly adenomas. ${ }^{24,25}$

While unidentified pathogenic factors are likely responsible for the greater frequency of invasion seen among firmer adenomas, the association between greater EOR and lower consistency grade is likely technical in origin. Due to more straightforward manipulation and resection of these tumors, there is a lower chance of residual or inaccessible disease. Furthermore, less invasive lesions are easier to resect in their entirety. The observation that consistency correlates with EOR nicely mirrors our prior work on meningiomas, and corroborates the skull base principle that lower-consistency-grade tumors are more resectable. ${ }^{26-28}$

The impetus for our descriptive 5-point grading scale derives from a noted trend in neuroimaging studies that use binary and subjective categories such as "soft" and "hard" based on retrospectively gathered data from neurosurgical operative reports. Furthermore, the radiology literature is full of conflicting reports on successful preoperative predictors of intraoperative consistency, which may include T2 intensity and/or diffusion-weighted imaging characteristics, but remains largely unproven and without clear clinical utility at present. ${ }^{6-8,25,29-31}$ We hope that the increased granularity of our grading scheme predisposes toward more accurate identification of radiological characteristics associated with adenoma consistency, and that the correlation between consistency and clinical outcomes highlights the importance of nonbinary grading that is objective. 


\section{Limitations}

The present study is not without limitations. Our objective was designed only to validate the clinical correlation between tumor consistency, as defined by our proposed category, with EOR, perioperative course, and complications. Future studies will aim to relate the intraoperative consistency scores to preoperative MRI studies in order to predict tumor consistency from imaging characteristics alone, allowing a more accurate estimation of the EOR and expected clinical course during the preoperative planning stage.

\section{Conclusions}

We have established a universally applicable, standardized, and easy-to-implement consistency grading scale for PAs. Clinical validation will come from prospective application to larger patient cohorts, but initial results indicate correlations with sex, functionality, invasion, EOR, and endocrinopathy. Future work may establish more definitive neuroimaging correlates following further application of our more granular consistency score.

\section{References}

1. Juraschka K, Khan OH, Godoy BL, et al. Endoscopic endonasal transsphenoidal approach to large and giant pituitary adenomas: institutional experience and predictors of extent of resection. J Neurosurg. 2014;121(1):75-83.

2. Cappabianca P, Cavallo LM, Esposito F, et al. Extended endoscopic endonasal approach to the midline skull base: the evolving role of transsphenoidal surgery. Adv Tech Stand Neurosurg. 2008;33:151-199.

3. de Divitiis E, Cavallo LM, Cappabianca P, Esposito F. Extended endoscopic endonasal transsphenoidal approach for the removal of suprasellar tumors: Part 2. Neurosurgery. 2007;60(1):46-59.

4. Theodosopoulos PV, Leach J, Kerr RG, et al. Maximizing the extent of tumor resection during transsphenoidal surgery for pituitary macroadenomas: can endoscopy replace intraoperative magnetic resonance imaging? J Neurosurg. 2010;112(4):736-743.

5. Yamamoto J, Kakeda S, Shimajiri S, et al. Tumor consistency of pituitary macroadenomas: predictive analysis on the basis of imaging features with contrast-enhanced 3D FIESTA at 3T. AJNR Am J Neuroradiol. 2014;35(2):297-303.

6. Pierallini A, Caramia F, Falcone C, et al. Pituitary macroadenomas: preoperative evaluation of consistency with diffusion-weighted MR imaging-initial experience. Radiology. 2006;239(1):223-231.

7. Yao A, Pain M, Balchandani P, Shrivastava RK. Can MRI predict meningioma consistency?: a correlation with tumor pathology and systematic review. Neurosurg Rev. 2018;41(3):745-753.

8. Zeynalova A, Kocak B, Durmaz ES, et al. Preoperative evaluation of tumour consistency in pituitary macroadenomas: a machine learning-based histogram analysis on conventional T2-weighted MRI. Neuroradiology. 2019;61(7):767-774.

9. Knosp E, Steiner E, Kitz K, Matula C. Pituitary adenomas with invasion of the cavernous sinus space: a magnetic resonance imaging classification compared with surgical findings. Neurosurgery. 1993;33(4):610-618.

10. Badie B, Brooks N, Souweidane MM. Endoscopic and minimally invasive microsurgical approaches for treating brain tumor patients. J Neurooncol. 2004;69(1-3):209-219.

11. Jankowski R, Auque J, Simon C, et al. Endoscopic pituitary tumor surgery. Laryngoscope. 1992;102(2):198-202.
12. Snow RB, Lavyne MH, Lee BC, et al. Craniotomy versus transsphenoidal excision of large pituitary tumors: the usefulness of magnetic resonance imaging in guiding the operative approach. Neurosurgery. 1986;19(1):59-64.

13. Itamura K, Chang K-E, Lucas J, et al. Prospective clinical validation of a meningioma consistency grading scheme: association with surgical outcomes and extent of tumor resection. J Neurosurg. 2019;131(5):1356-1360.

14. Zada G, Yashar P, Robison A, et al. A proposed grading system for standardizing tumor consistency of intracranial meningiomas. Neurosurg Focus. 2013;35(6):E1.

15. Jagannathan J, Smith R, DeVroom HL, et al. Outcome of using the histological pseudocapsule as a surgical capsule in Cushing disease. J Neurosurg. 2009;111(3):531-539.

16. Kawamata T, Kubo O, Hori T. Surgical removal of growth hormone-secreting pituitary adenomas with intensive microsurgical pseudocapsule resection results in complete remission of acromegaly. Neurosurg Rev. 2005;28(3):201-208.

17. Kim EH, Ku CR, Lee EJ, Kim SH. Extracapsular en bloc resection in pituitary adenoma surgery. Pituitary. 2015;18(3):397-404.

18. Monteith SJ, Starke RM, Jane JA Jr, Oldfield EH. Use of the histological pseudocapsule in surgery for Cushing disease: rapid postoperative cortisol decline predicting complete tumor resection. J Neurosurg. 2012;116(4):721-727.

19. Xie T, Liu T, Zhang X, et al. Time to revive the value of the pseudocapsule in endoscopic endonasal transsphenoidal surgery for growth hormone adenomas. World Neurosurg. 2016;89:65-71.

20. Thotakura AK, Patibandla MR, Panigrahi MK, Mahadevan A. Is it really possible to predict the consistency of a pituitary adenoma preoperatively? Neurochirurgie. 2017;63(6):453457.

21. Jahangiri A, Wagner JR, Chin AT, et al. Incidence of headache as a presenting complaint in over 1000 patients with sellar lesions and factors predicting postoperative improvement. Clin Neurol Neurosurg. 2015;132:16-20.

22. Wolf A, Goncalves S, Salehi F, et al. Quantitative evaluation of headache severity before and after endoscopic transsphenoidal surgery for pituitary adenoma. J Neurosurg. 2016;124(6):1627-1633.

23. Yagnik G, Rutowski MJ, Shah SS, Aghi MK. Stratifying nonfunctional pituitary adenomas into two groups distinguished by macrophage subtypes. Oncotarget. 2019;10(22):22122223.

24. Zada G, Lin N, Laws ER Jr. Patterns of extrasellar extension in growth hormone-secreting and nonfunctional pituitary macroadenomas. Neurosurg Focus. 2010;29(4):E4.

25. Wei L, Lin SA, Fan K, et al. Relationship between pituitary adenoma texture and collagen content revealed by comparative study of MRI and pathology analysis. Int J Clin Exp Med. 2015;8(8):12898-12905.

26. Little KM, Friedman AH, Sampson JH, et al. Surgical management of petroclival meningiomas: defining resection goals based on risk of neurological morbidity and tumor recurrence rates in 137 patients. Neurosurgery. 2005;56(3):546-559.

27. Sitthinamsuwan B, Khampalikit I, Nunta-aree S, et al. Predictors of meningioma consistency: a study in 243 consecutive cases. Acta Neurochir(Wien). 2012;154(8):1383-1389.

28. Stafford SL, Perry A, Suman VJ, et al. Primarily resected meningiomas: outcome and prognostic factors in 581 Mayo Clinic patients, 1978 through 1988. Mayo Clin Proc. 1998;73:936-942.

29. Hughes JD, Fattahi N, Van Gompel J, et al. Magnetic resonance elastography detects tumoral consistency in pituitary macroadenomas. Pituitary. 2016;19(3):286-292.

30. Sanei Taheri M, Kimia F, Mehrnahad M, et al. Accuracy of diffusion-weighted imaging-magnetic resonance in differentiating functional from non-functional pituitary macro- 
adenoma and classification of tumor consistency. Neuroradiol J. 2019;32(2):74-85.

31. Smith KA, Leever JD, Chamoun RB. Prediction of consistency of pituitary adenomas by magnetic resonance imaging. J Neurol Surg B Skull Base. 2015;76(5):340-343.

\section{Disclosures}

The authors report no conflict of interest concerning the materials or methods used in this study or the findings specified in this paper.

\section{Author Contributions}

Conception and design: Rutkowski, Chang, Carmichael, Zada. Acquisition of data: Cardinal, Brunswick, Micko. Analysis and interpretation of data: Rutkowski, Brunswick, Micko, Liu, Shiroishi. Drafting the article: Rutkowski, Chang. Critically revising the article: Rutkowski, Du, Tafreshi, Donoho, Liu, Shiroishi,
Carmichael, Zada. Reviewed submitted version of manuscript: Rutkowski, Cardinal, Donoho, Liu, Shiroishi, Carmichael, Zada. Approved the final version of the manuscript on behalf of all authors: Rutkowski. Statistical analysis: Rutkowski, Micko. Study supervision: Zada.

\section{Supplemental Information}

Videos

Video 1. https://vimeo.com/405796085.

\section{Correspondence}

Martin J. Rutkowski: University of Southern California, Los Angeles, CA. martin.rutkowski@gmail.com. 